MR IMRAN H. YUSUF (Orcid ID : 0000-0002-0424-5852)

Article type : Letter to the Editor

\title{
Joint recommendations for retinal screening in long-term users of hydroxychloroquine and chloroquine in the United Kingdom (2018).
}

\author{
I. H. Yusuf ${ }^{1}$, A. J. Lotery*,2 M.R. Ardern-Jones*3
}

(1) Imran H. Yusuf MB ChB (Hons), MRes, MRCP(UK), FRCOphth; imran.yusuf@merton.ox.ac.uk

(2) Andrew J. Lotery MD, FRCOphth; A.J.Lotery@soton.ac.uk

(3) Michael R. Ardern-Jones BSc, MBBS, DPhil, FRCP; m.aj@soton.ac.uk

* Joint corresponding authors: Michael R. Arden-Jones; m.aj@soton.ac.uk \& Andrew J. Lotery; A.J.Lotery@soton.ac.uk

(1) The Oxford Eye Hospital, West Wing, John Radcliffe Hospital, Headley Way, Headington, Oxford, OX3 9DU

(2) Clinical and Experimental Sciences, Faculty of Medicine, University of Southampton, Southampton, S016 6YD

(3) Department of Dermatology, University Hospital Southampton NHS Foundation Trust, Tremona Road, Southampton, Hampshire, S016 6YD

This article has been accepted for publication and undergone full peer review but has not been through the copyediting, typesetting, pagination and proofreading process, which may lead to differences between this version and the Version of Record. Please cite this article as doi:

$10.1111 /$ bjd. 16782

This article is protected by copyright. All rights reserved. 
None of the authors have any financial interest in a product, method or material or lack thereof.

$B J D$

Manuscript word count (750): 750

References (8): 8

Hydroxychloroquine has become established in the management of various forms of cutaneous lupus, photosensitive dermatoses, porphyria cutanea tarda, cutaneous sarcoid, lichen planus and other inflammatory dermatological diseases. ${ }^{1}$ Dermatologists who prescribe hydroxychloroquine should be aware of new recommendations for retinal screening in all patients on long-term hydroxychloroquine and chloroquine in the United Kingdom. ${ }^{2}$ The recommendations have been published by the Royal College of Ophthalmologists following a systematic review of the current evidence with input from representatives from the British Society for Rheumatology (BSR) ${ }^{3}$ and the British Association of Dermatologists (BAD) (Dr. M Ardern-Jones). The recommendations do not apply to mepacrine or quinacrine.

Hydroxychloroquine can cause retinal degeneration with long-term use. ${ }^{4}$ Hydroxychloroquine retinopathy only becomes symptomatic once the retinal degeneration is advanced, manifesting at that stage as bilateral, irreversible visual loss. Modern retinal imaging techniques (optical coherence tomography and fundus autofluoresence imaging) together with central visual field testing are able to detect hydroxychloroquine retinopathy at an early, pre-symptommatic stage. ${ }^{5}$ With such techniques, the prevalence of retinopathy has been estimated at around $7.5 \%$ in patients taking hydroxychloroquine for more than 5 years, increasing to $20-50 \%$ in patients taking hydroxychloroquine for more than 20 years. $^{5}$

The increasing use of hydroxychloroquine within the U.K., ${ }^{2}$ increasing numbers of patients presenting to eye clinics with severe hydroxychloroquine retinopathy, recognition of sporadic/opportunistic retinal monitoring in motivated patients, the use of varying screening protocols, the evidence demonstrating a higher prevalence of retinal toxicity in long-term users and the widespread availability of retinal imaging technology have supported the case for screening for hydroxychloroquine retinopathy. ${ }^{2}$

This article is protected by copyright. All rights reserved. 
The new guideline recommends that all patients taking hydroxychloroquine or chloroquine where long-term treatment is planned ( $>5$ years for hydroxychloroquine and $>1$ year for chloroquine) should receive a baseline evaluation of retinal structure and function, ideally within 6 months of commencing treatment (definitely within 12 months). ${ }^{2}$ All individuals who have taken hydroxychloroquine for greater than 5 years should receive annual screening for retinopathy. ${ }^{2}$ Individuals with additional risk factors for hydroxychloroquine retinopathy should be screened after 1 year of therapy. Additional risk factors for retinopathy include concomitant tamoxifen use (odds ratio - 4.59), impaired renal function (estimated glomerular filtration rate of less than $60 \mathrm{ml} / \mathrm{min} / 1.73 \mathrm{~m}^{2}$ odds ratio -2.08 ), or daily dose in excess of $5 \mathrm{mg} / \mathrm{kg}$ per day of hydroxychloroquine (odds ratio - 5.67)..$^{5}$ Chloroquine is considered more toxic to the retina than hydroxychloroquine: patients on chloroquine should be screened annually after 1 year of drug therapy.

Baseline evaluation and screening tests will be undertaken in the local hospital eye service and will include retinal imaging and visual field testing. The results of screening will be communicated to the patient, GP and prescribing physician as either "no retinopathy", "possible retinopathy" or "definite retinopathy". The recommendations state that patients with "possible retinopathy" should continue drug treatment. In view of the slow disease course of retinopathy in these cases, the recommendation to stop hydroxychloroquine in patients with "definite retinopathy" will be made by the ophthalmologist to the prescribing physician for assimilation of risk. Screening for hydroxychloroquine retinopathy will terminate if a patient stops taking hydroxychloroquine, or if definite retinopathy is detected.

Patient education is important in helping patients to understand the risks of long-term hydroxychloroquine treatment, the purpose of retinal screening, and the likely screening schedule. A patient information leaflet has been produced by the Macular Society with input from the RCOphth on screening for hydroxychloroquine retinopathy. ${ }^{6}$ This leaflet should be given to all patients taking hydroxychloroquine or chloroquine, and ideally at treatment initiation for new users. The date at which screening should be initiated (e.g. 5 years after starting hydroxychloroquine), cannot be coordinated by hospital eye services and will be the responsibility of patients and prescribing physicians.

This article is protected by copyright. All rights reserved. 
It is worth noting that General Medical Council guidance suggests that the responsibility for initiating screening rests with the prescribing physician. It would seem appropriate that specialists communicate the need for screening at the appropriate time interval to primary care physicians who take on the prescribing role. The RCOphth have provided a referral proforma attached as an Appendix to the recommendations on hydroxychloroquine retinopathy. $^{2}$

We suggest that dermatologists who prescribe hydroxychloroquine become familiar with the joint recommendations for screening for hydroxychloroquine retinopathy in the United Kingdom. ${ }^{2}$ The estimated prevalence of hydroxychloroquine use in the United Kingdom predicts a large number of patients who require annual screening. ${ }^{7}$ The demands of the guidelines are likely to present challenges in establishing appropriate referral pathways and virtual clinics in local eye units. ${ }^{8}$ However. systematic retinal screening should reduce the risk of avoidable and irreversible blindness from hydroxychloroquine toxicity.

\section{References}

1. Templeman R, Piliouras P, Robertson I. A review of antimalarial agents in dermatology. Australasian Journal of Dermatology 2014;55:24.

2. Royal College of Ophthalmologists Guideline Development Group - Hydroxychloroquine and Chloroquine Retinopathy: Recommendations on Screening [https://www.rcophth.ac.uk/standards-publications-research/clinical-guidelines/ - Accessed on 3rd March 2018]. 2018.

3. Yusuf IH, Ledingham JM, MacPhie E, et al. Monitoring for retinal toxicity in patients taking hydroxychloroquine and chloroquine. Rheumatology (Oxford, England) 2018.

4. Yusuf IH, Sharma S, Luqmani R, Downes SM. Hydroxychloroquine retinopathy. Eye (London, England) 2017;31:828-45.

This article is protected by copyright. All rights reserved. 
5. Melles RB, Marmor MF. The risk of toxic retinopathy in patients on long-term hydroxychloroquine therapy. JAMA ophthalmology 2014;132:1453-60.

6. Eye screening for patients taking hydroxychloroquine (Plaquenil@): Macular Society $\begin{array}{lllll}\text { [Accessed } & \text { on } & \text { 1st } & \text { March }\end{array}$ https://www.macularsociety.org/sites/default/files/resource/Hydroxychloroquine\%20\%20access.pdf]. 2018.

7. Yates M, Malaiya R, Stack J, Galloway JB. Hydroxychloroquine use: the potential impact of new ocular screening guidelines. Eye (London, England) 2018;32:161-2.

8. Tucker WR, Galloway J, Walsh S. The gathering storm: hydroxychloroquine retinopathy screening in the U.K. The British journal of dermatology 2017;176:1420-1.

This article is protected by copyright. All rights reserved. 